\title{
ROTATION OF THE BODY WITH MOVABLE INTERNAL MASSES AROUND THE CENTER OF MASS ON THE ROUGH PLANE
}

\author{
ALEKSANDR SAKHAROV \\ Moscow Institute of Physics and Technology (State University) \\ 9 Institutskiy per., Dolgoprudny, Moscow Region, Russian Federation \\ e-mail: sah.aleksandr@gmail.com, web page: http://www.mipt.ru/
}

Key words: Dry Friction, Combined Friction, Normal Stress, Multibody System, Movable Masses, Slider

\begin{abstract}
We consider motion of a system consisting of a rigid body and internal movable masses on a rough surface. The possibility of rotation of the system around its center of mass because of the internal movable mass motion is investigated. To describe the friction between the body and the reference surface selected local Amonton-Coulomb law. To determine the distribution of the normal stress in the contact area between the body and the surface linear dynamically collaborative model is used. As examples we consider two configurations of internal masses: hard horizontal disk and two material points, moving parallel to the longitudinal axis of the body symmetry in the opposite way. Motions of the system analyzing for selected configurations.
\end{abstract}

\section{INTRODUCTION}

At the moment mobile robotics find wide application in various fields of human activity: research, medicine, space, etc. Among the large variety of robotic systems moving on a plane, class of the devices which move without any external moving parts, such as wheels, tracks, legs can be distinguished. Such systems have a number of advantages associated with their hermiticity and isolation from external adverse factors. For example a robotball could be called.

Motion of the robot without external drivers can be achieved by using movable internal masses and interaction with the supporting surface by friction forces. In the works $[1,2]$ investigated one-dimensional motion of a dual-mass system in the presence of dry friction forces. Optimal parameters which give the highest average speed of the body with imposed restrictions is determined. In the paper [3] investigated the body with one and two internal masses and three models of resistance to the robot motion: a piecewise-linear friction, quadratic friction and dry friction. 
Two-dimensional movement of the three-mass robot in a liquid medium is investigated in the work $[4,5]$. The motion of the system is achieved by moving two internal masses and the forces of viscous resistance. The possibility of the body rotation on a rough surface with the rotor inside the body, which rapidly rotating around the longitudinal axis of the body symmetry while the body move direct this axis is proved in $[6,7]$.

In this work, we study the rotation of the symmetrical hollow body around its center of mass with by moving material points inside the body. It is assumed that the points are located on a horizontal plane passing through the body center of mass and system center of mass does not move inside the body. In contrast to $[6,7]$, in the present work applies the local friction of rest.

\section{MODEL OF THE SYSTEM}

Consider a rigid body of mass $m_{0}$, representing a hollow parallelepiped composed of six homogeneous rectangles. Length of the body equal to $a$, width $-b$, height $-2 h$. The body relies on a rough horizontal surface. We introduce a laboratory coordinates system $O^{\prime} x y z$ with start on the surface and connected with the body system $O \xi \eta \zeta$, where $O$ - body center of mass (Fig. 1). Axis $O^{\prime} z$ and $O \zeta$ forward vertically, the axis $O \xi$ is parallel to the larger edge of the parallelepiped base, the axis $O \eta$ directed so that the system formed right-hand triple. We suppose that in the initial moment corresponding axis coincide. The main axis of the central ellipsoid of inertia of the body coincide with the coordinates system $O \xi \eta \zeta$. Moment of inertia with respect to axis $O \zeta$ is equal to $C$. Inside the body material points are located such way that center of mass of system coincides with the center of mass of the body $O$. The system consists of the body and internal masses will called a slider.

In the general case position of the body is determined by three coordinates. Coordinates $x_{O}, y_{O}$ determine point $O$ in the laboratory coordinates system. The angle $\varphi$ between the axes $O^{\prime} x$ and $O \xi$ determine rotation of the slider relative to its initial position. But in this work we suppose that at the initial time body stay at rest. Due to the special chose of the internal mass configuration and motion point $O$ will be fixed during the whole time of motion. Thus position of the body is determined by the angle of rotation $\varphi$ and coordinates of points $O^{\prime}$ and $O$ are $x_{O}=x_{O^{\prime}}=0, y_{O}=y_{O^{\prime}}=0, z_{O}=h, z_{O^{\prime}}=0$.

\section{FORCES AND MOMENTS}

Let $\sum_{i=1}^{s} m_{i}$ - is the sum of the inside movable material points masses, $s$ - the number of points. Write theorem on the slider center of mass motion and the momentum change of the slider relative to the point $O$ :

$$
\begin{gathered}
m \ddot{\boldsymbol{r}}_{O}=\boldsymbol{F}_{g}+\boldsymbol{F}_{n}+\boldsymbol{F}_{t}, \quad m=m_{0}+\sum_{i=1}^{s} m_{i}, \\
\dot{\boldsymbol{K}}_{O}=\boldsymbol{M}_{O}^{g}+\boldsymbol{M}_{O}^{n}+\boldsymbol{M}_{O}^{t},
\end{gathered}
$$




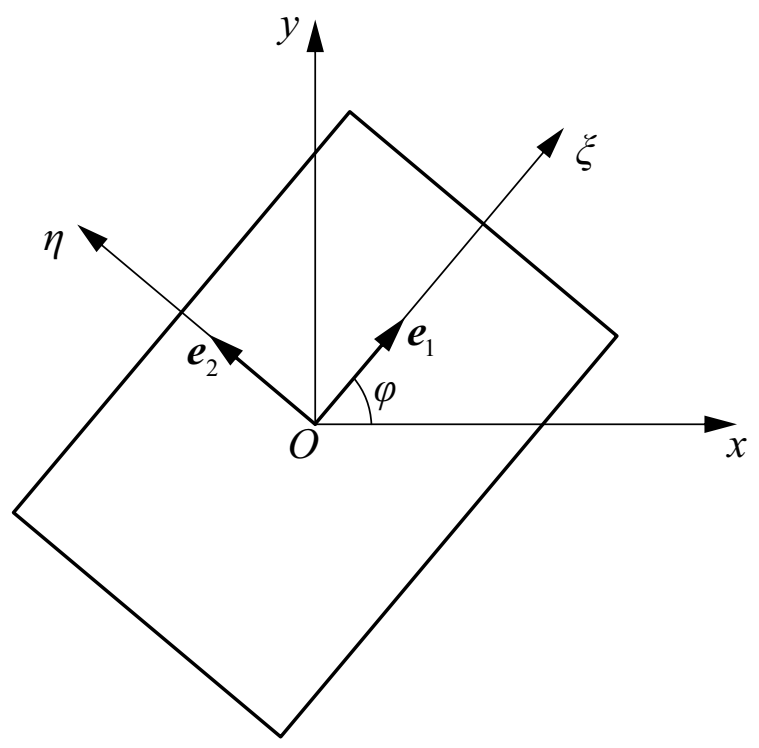

Figure 1: Laboratory and body systems of coordinates

where $\boldsymbol{r}_{O}=\overrightarrow{O^{\prime} O}$ - the radius-vector directed from the point $O^{\prime}$ to the body center of mass $O, \boldsymbol{F}_{g}$ - the force of gravity acting on the slider, $\boldsymbol{F}_{n}$ and $\boldsymbol{F}_{t}$ - the main vectors of norm reactions and friction forces respectively. $\boldsymbol{K}_{O}$ - kinetic moment of the slider relative the point $O, \boldsymbol{M}_{O}^{g}$ - the main moment of gravity force relative to the point $O, \boldsymbol{M}_{O}^{n}$ and $\boldsymbol{M}_{O}^{t}{ }^{-}$ the main moment of normal reactions and friction forces relative the point $O$ respectively.

The radius-vector $\boldsymbol{r}_{O}$ and unit vectors of the body coordinates system is equal to

$$
\boldsymbol{r}_{O}=\left[\begin{array}{l}
0 \\
0 \\
h
\end{array}\right], \quad \boldsymbol{e}_{1}=\left[\begin{array}{c}
\cos \varphi \\
\sin \varphi \\
0
\end{array}\right], \quad \boldsymbol{e}_{2}=\left[\begin{array}{c}
-\sin \varphi \\
\cos \varphi \\
0
\end{array}\right], \quad \boldsymbol{k}=\left[\begin{array}{l}
0 \\
0 \\
1
\end{array}\right],
$$

Kinetic moment of the slider $\boldsymbol{K}_{O}$ can be represented as a sum of the kinetic moments of the body and internal movable masses:

$$
\boldsymbol{K}_{O}=\boldsymbol{K}_{O 0}+\sum_{i=1}^{s} \boldsymbol{K}_{O i}
$$

where $\boldsymbol{K}_{O 0}=C \dot{\varphi} \boldsymbol{k}$ - kinetic moment of the body, $\boldsymbol{K}_{O i}$ - kinetic moment of $i$-th internal mass.

The force of gravity, main vectors of normal reactions and friction forces, as well as the corresponding moments are determined by the following expression:

$$
\begin{gathered}
\boldsymbol{F}_{g}=-m g \boldsymbol{k}, \quad \boldsymbol{F}_{n}=\boldsymbol{k} \iint n_{A} d s, \quad \boldsymbol{F}_{t}=\iint \boldsymbol{t}_{A} d s \\
\boldsymbol{M}_{O}^{g}=-\sum_{i=1}^{s} \boldsymbol{r}_{O i} \times m_{i} g \boldsymbol{k}, \quad \boldsymbol{M}_{O}^{n}=\iint n_{A} \boldsymbol{r}_{O A} \times \boldsymbol{k} d s, \quad \boldsymbol{M}_{O}^{t}=\iint \boldsymbol{r}_{O A} \times \boldsymbol{t}_{A} d s .
\end{gathered}
$$


Here and elsewhere the integration extends over the region $D(\xi, \eta)$, consisting of body and surface contact points. $n_{A}$ and $\boldsymbol{t}_{A}$ - normal and tangential stresses at point $A \in D$, $\boldsymbol{r}_{O i}=\xi_{i}(t) \boldsymbol{e}_{1}+\eta_{i}(t) \boldsymbol{e}_{2}$ and $\boldsymbol{r}_{O A}=\xi \boldsymbol{e}_{1}+\eta \boldsymbol{e}_{2}-h \boldsymbol{k}$ - radius-vectors from the point $\mathrm{O}$ to the $i$-th internal mass and point $A$, respectively. Friction is locally described by the locally Amontons-Coulomb law, which extend to the case of the body at rest:

$$
\boldsymbol{t}_{A}=-\mu n_{A} \boldsymbol{f}_{A}, \quad \boldsymbol{f}_{A}= \begin{cases}v_{A}^{-1} \boldsymbol{v}_{A}, & v_{A} \neq 0 \\ \boldsymbol{\tau}_{A}, & v_{A}=0\end{cases}
$$

where $\mu$-coefficient of friction, $\boldsymbol{v}_{A}=\dot{\boldsymbol{r}}_{A}, \boldsymbol{r}_{A}=\overrightarrow{O^{\prime} A}$. Since $\boldsymbol{v}_{O}=0$, velocity of the arbitrary point $A$ of the slider base express by the formula:

$$
\boldsymbol{v}_{A}=\dot{\varphi} \boldsymbol{k} \times \boldsymbol{r}_{O A}=\dot{\varphi}\left(\xi \boldsymbol{e}_{2}-\eta \boldsymbol{e}_{1}\right),
$$

where $\dot{\varphi} \boldsymbol{k}$ - angular velocity of the body. Thus, unit vector $v_{A}^{-1} \boldsymbol{v}_{A}$ expressed:

$$
v_{A}^{-1} \boldsymbol{v}_{A}=\frac{\dot{\varphi}}{|\dot{\varphi}|} \frac{-\eta \boldsymbol{e}_{1}+\xi \boldsymbol{e}_{2}}{\sqrt{\xi^{2}+\eta^{2}}} .
$$

The module of the vector $\boldsymbol{\tau}_{A}$ is determined by attached to the slider forces and not greater than one. This approach was previously proposed by John H. Jellett $[8,9]$. Because point $O$ fixed, vectors $\boldsymbol{v}_{A}$ directed perpendicular to the radius-vector $\boldsymbol{r}_{O A}$. Suppose that vectors $\boldsymbol{\tau}_{A}$ have the same orientation. Combining vectors $v_{A}^{-1} \boldsymbol{v}_{A}$ and $\boldsymbol{\tau}_{A}$ in one formula we obtain the following expression (Fig. 2):

$$
\boldsymbol{f}_{A}=f_{\xi} \boldsymbol{e}_{1}+f_{\eta} \boldsymbol{e}_{2}, \quad f_{\xi}=-f_{A} \frac{\eta}{\sqrt{\xi^{2}+\eta^{2}}}, \quad f_{\eta}=f_{A} \frac{\xi}{\sqrt{\xi^{2}+\eta^{2}}}, \quad-1 \leq f_{A} \leq 1 .
$$

Continuity condition of the contact between the body and the surface imposes on the kinematic characteristics three independent constraints. Therefore, the model of normal stresses $n_{A}$ should include three independent parameters $\lambda_{0}, \lambda_{1}$ and $\lambda_{2}$, which is determines from these limitations each time. This model of normal stress distribution is dynamically collaborative $[8,10]$.

$$
n_{A}=\lambda_{0}+\lambda_{1} \xi+\lambda_{2} \eta, \quad(\xi, \eta) \in D .
$$

The physical interpretation of this formula can serve as a representation of small deformations of the surface in the contact area, leading to the normal stresses by Hooke's law.

Substituting equation expressing the Amontons-Coulomb law and formula (6) in expressions (4), (5), then substituting the resulting expression into a system (1), (2), we get the equation of motion of the slider in closed form. Each time are unknown coordinate $\varphi$ (coordinates $x_{O}, y_{O}$ assumed to be constant) and the coefficients $\lambda_{0}, \lambda_{1}$ and $\lambda_{2}$ of the linear model (6). 


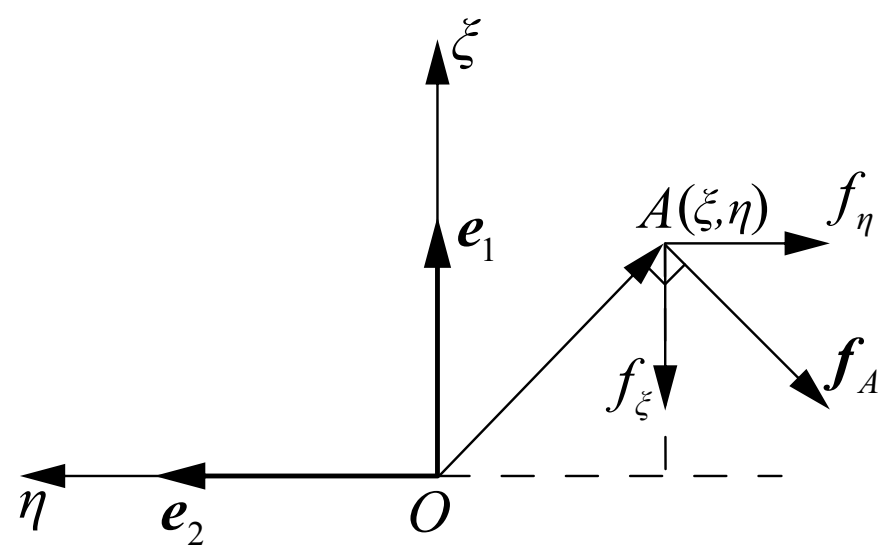

Figure 2: Decomposition of function $\boldsymbol{f}_{A}$ on the basis $\boldsymbol{e}_{1}, \boldsymbol{e}_{2}$

\section{COEFFICIENTS OF THE NORMAL STRESS MODEL}

To determine the coefficient $\lambda_{0}$ in the decomposition (6), simplify the expression for the main vector of normal reaction calculating the integral in the second expression of the (4). For this purpose it is necessary to take into account the symmetry of the slider and surface contact area $D(\xi, \eta)$ :

$$
-\frac{a}{2} \leq \xi \leq \frac{a}{2}, \quad-\frac{b}{2} \leq \eta \leq \frac{b}{2}
$$

Then the second expression of the (4) is easily integrated:

$$
\boldsymbol{F}_{n}=\boldsymbol{k} \iint\left(\lambda_{0}+\lambda_{1} \xi+\lambda_{2} \eta\right) d \xi d \eta=\lambda_{0} a b \boldsymbol{k} .
$$

The continuity condition of the contact between the slider and the surface is equal to zero projections of the velocities (and accelerations) of the body points on the vertical. This condition can be expressed in three independent equations. First equation is obtained from the required that projection of the main force on the vertical equality to zero, i.e. the projection of the equation (1) on unit vector $\boldsymbol{k}$ :

$$
\left(\boldsymbol{F}_{g}+\boldsymbol{F}_{n}\right) \cdot \boldsymbol{k}=0 .
$$

Substituting first formula (4) and (7) in (8), we find the coefficient $\lambda_{0}$ :

$$
\lambda_{0}=\frac{m g}{a b} .
$$

Physical sense of the value $\lambda_{0}$ is the average pressure of the body weight $m g$ on a surface area $a b$.

To obtain expressions for the coefficients $\lambda_{1}$ and $\lambda_{2}$ we project equation (2) on unit vectors $\boldsymbol{e}_{j},(j=1,2)$. Main moment of the gravitational forces equals to zero because of 
the configuration symmetry of the internal masses: $\boldsymbol{M}_{O}^{g}=0$. For the projections of the main moments of the normal reactions and friction forces $\boldsymbol{M}_{O}^{n}$ and $\boldsymbol{M}_{O}^{t}$ we obtain:

$$
\begin{gathered}
\boldsymbol{M}_{O}^{n} \cdot \boldsymbol{e}_{j}=\iint n_{A}\left(\boldsymbol{r}_{O A} \times \boldsymbol{k}\right) \cdot \boldsymbol{e}_{j} d s=\iint n_{A} \boldsymbol{k} \cdot\left(\boldsymbol{e}_{j} \times \boldsymbol{r}_{O A}\right) d s, \\
\boldsymbol{M}_{O}^{t} \cdot \boldsymbol{e}_{j}=-\iint \mu n_{A}\left(\boldsymbol{r}_{O A} \times \boldsymbol{f}_{A}\right) \cdot \boldsymbol{e}_{j} d s=-\iint \mu n_{A} \boldsymbol{f}_{A} \cdot\left(\boldsymbol{e}_{j} \times \boldsymbol{r}_{O A}\right) d s .
\end{gathered}
$$

We also use the decomposition (6), consider that $\dot{\boldsymbol{K}}_{O} \cdot \boldsymbol{e}_{j}=0$ (all internal masses move at the horizontal plane containing point $O$ ) and then obtain a system of two equations:

$$
\iint \psi_{j}\left(\lambda_{0}+\lambda_{1} \xi+\lambda_{2} \eta\right) d s=0, \quad \psi_{j}=\left(\boldsymbol{k}-\mu \boldsymbol{f}_{A}\right) \cdot\left(\boldsymbol{e}_{j} \times \boldsymbol{r}_{O A}\right) .
$$

Perform calculations in the expression of $\psi_{j}$ to simplify it:

$$
\psi_{1}=\eta-\mu h f_{\eta}, \quad \psi_{2}=-\xi+\mu h f_{\xi} .
$$

We rewrite the expression (10) in the form of a two linear equations relative $\lambda_{1}$ and $\lambda_{2}$ :

$$
\begin{aligned}
& a_{11} \lambda_{1}+a_{12} \lambda_{2}=a_{10} \lambda_{0}, \\
& a_{21} \lambda_{1}+a_{22} \lambda_{2}=a_{20} \lambda_{0},
\end{aligned}
$$

where coefficients:

$$
\begin{array}{lll}
a_{11}=\iint \psi_{1} \xi d s, & a_{12}=\iint \psi_{1} \eta d s, & a_{10}=-\iint \psi_{1} d s \\
a_{21}=\iint \psi_{2} \xi d s, & a_{22}=\iint \psi_{2} \eta d s, & a_{20}=-\iint \psi_{2} d s .
\end{array}
$$

The solution of the system:

$$
\lambda_{1}=\frac{a_{10} a_{22}-a_{12} a_{20}}{a_{11} a_{22}-a_{12} a_{21}} \lambda_{0}, \quad \lambda_{2}=\frac{a_{11} a_{20}-a_{10} a_{21}}{a_{11} a_{22}-a_{12} a_{21}} \lambda_{0} .
$$

It is easy to verify that in this case $\lambda_{1}$ and $\lambda_{2}$ are equal to zero. Indeed, coefficients $a_{10}=a_{20}=0$ due to the odd of subintegral function $\psi_{j}$ and symmetry of the region $D$. It remains to show that the denominator in the formula (11) is not equal to zero:

$$
\begin{gathered}
a_{11} a_{22}=-(\mu h)^{2} \iint f_{\eta} \xi d s \iint f_{\xi} \eta d s= \\
=\left(\mu h f_{A}\right)^{2} \iint \frac{\xi^{2}}{\sqrt{\xi^{2}+\eta^{2}}} d s \iint \frac{\eta^{2}}{\sqrt{\xi^{2}+\eta^{2}}} d s>0, \\
a_{12} a_{21}=\left(\frac{a b^{3}}{12}-\mu h \iint f_{\eta} \eta d s\right)\left(-\frac{a^{3} b}{12}+\mu h \iint f_{\xi} \xi d s\right)=-\frac{a^{4} b^{4}}{144}<0 .
\end{gathered}
$$

It follows that

$$
a_{11} a_{22}-a_{12} a_{21}>0 .
$$

Thus, in this case, we find that $\lambda_{1}=\lambda_{2}=0$. Within the selected linear model, the distribution of the contact stresses is constant: $n_{A}=\lambda_{0}$. 


\section{EQUATION OF MOTION}

To obtain the equation of the slider motion projecting equation (2) on the unit vector $k$ :

$$
\dot{\boldsymbol{K}}_{O} \cdot \boldsymbol{k}=\boldsymbol{M}_{O}^{t} \cdot \boldsymbol{k}
$$

Discover the right part of this equation:

$$
\boldsymbol{M}_{O}^{t} \cdot \boldsymbol{k}=-\mu \iint n_{A}\left[\left(\xi \boldsymbol{e}_{1}+\eta \boldsymbol{e}_{2}-h \boldsymbol{k}\right) \times \boldsymbol{f}_{A}\right] \cdot \boldsymbol{k} d s=-\mu \lambda_{0} \iint\left(f_{\eta} \xi-f_{\xi} \eta\right) d s .
$$

Substituting this expression, formula (3) and expressions for $f_{\xi}, f_{\eta}$ in equation (12), we obtain following equation of motion

$$
C \ddot{\varphi}+\sum_{i=1}^{s} \dot{\boldsymbol{K}}_{O i} \cdot \boldsymbol{k}=-f_{A} T_{\varphi},
$$

where

$$
T_{\varphi}=\mu \lambda_{0} \iint \sqrt{\xi^{2}+\eta^{2}} d s
$$

Left part of this equation depends on the specific configurations of the internal masses and the laws of their relative motion. Let us consider two examples.

\subsection{Disk}

Let us put inside the body a disk mass $m_{d}$ and radius $d$ with horizontal plane and the centre coincided with the point $O$ (Figure 3a). Assume that the disk could rotate around its axis by engine. Then its kinetic moment equals to:

$$
\sum_{i=1}^{s} \boldsymbol{K}_{O i}=\boldsymbol{K}_{O d}=J_{d}\left(\dot{\varphi}+\omega_{d}\right) \boldsymbol{k},
$$

where $J_{d}=\frac{m_{d} d^{2}}{2}-$ axial moment of inertia, $\omega_{d} \boldsymbol{k}-$ angular velocity of the disk relative to the body.

Substituting this formula into equation (13), we obtain the equation of the slider motion with the horizontal disk located inside:

$$
\left(C+J_{d}\right) \ddot{\varphi}=R_{d}-f_{A} T_{\varphi},
$$

where $R_{d}=-J_{d} \dot{\omega}_{d}$. Considering the double inequality $-1 \leq f_{A} \leq 1$, the value $f_{A}$ is defined by following expression:

$$
f_{A}= \begin{cases}\dot{\varphi}|\dot{\varphi}|^{-1}, & \dot{\varphi} \neq 0, \\ R_{d} T_{\varphi}^{-1}, & \dot{\varphi}=0,\left|R_{d}\right| \leq T_{\varphi}, \\ R_{d}\left|R_{d}\right|^{-1}, & \dot{\varphi}=0,\left|R_{d}\right|>T_{\varphi} .\end{cases}
$$




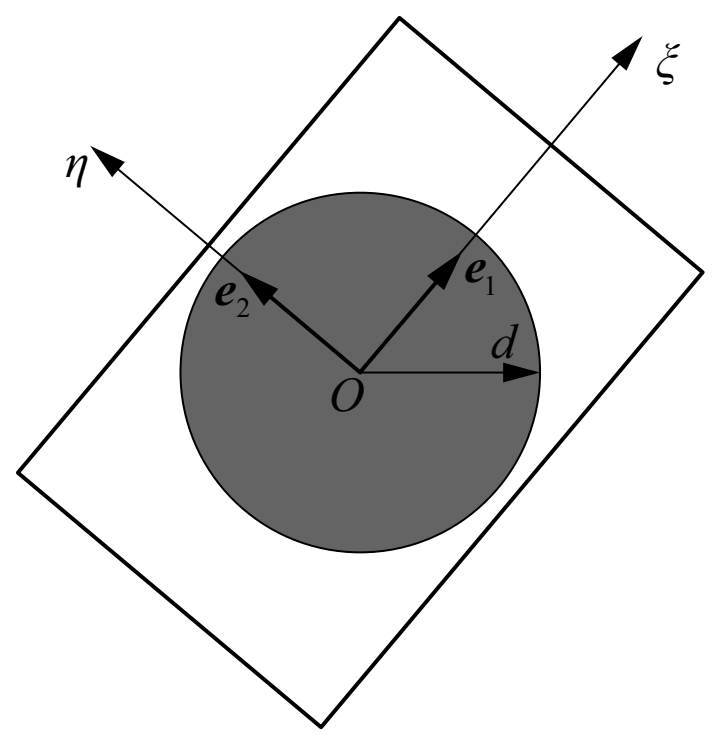

(a)

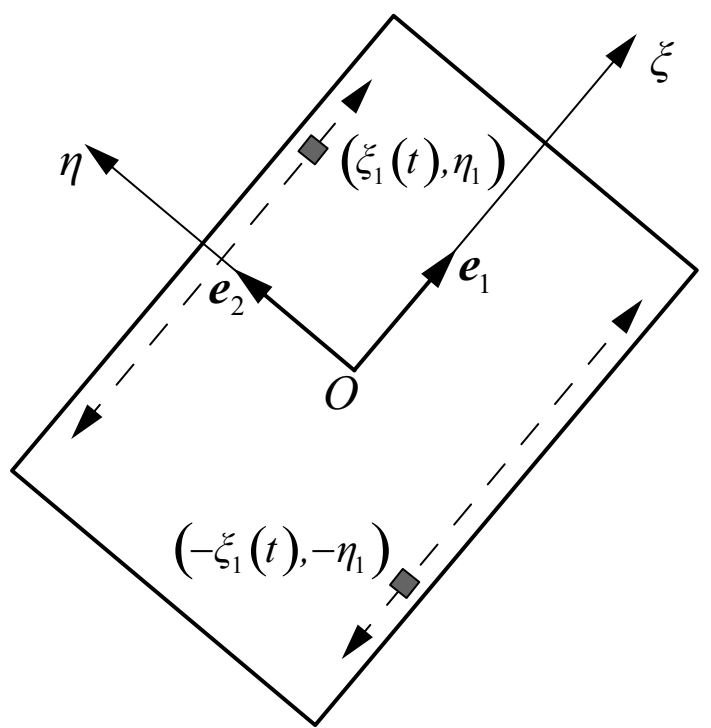

(b)

Figure 3: Slider with the disk (a) and two point masses (b)

From this formula is possible to find the minimum value of angular acceleration of the rotor $\dot{\omega}_{d}$ is required to start rotation of the body:

$$
\left|\dot{\omega}_{d}\right|>\frac{T_{\varphi}}{J_{d}}
$$

As an example of a simple control law of the relative angular acceleration of the disk we choose the two-phase piecewise constant periodic function. Then the relative angular velocity of the disc must satisfy the periodicity conditions: $\omega_{d}(0)=\omega_{d}\left(t^{*}\right)=0$, where $t^{*}$ is the period of the disk rotation. Such conditions the following function satisfies:

$$
\omega_{d}=\Omega \cdot\left\{\begin{array}{ll}
t \tau^{-1}, & 0 \leq t<\tau, \\
\left(t-t^{*}\right)\left(\tau-t^{*}\right)^{-1}, & \tau \leq t \leq t^{*} ;
\end{array} \quad \dot{\omega}_{d}=\Omega \cdot \begin{cases}\tau^{-1}, & 0 \leq t<\tau, \\
\left(\tau-t^{*}\right)^{-1}, & \tau \leq t \leq t^{*}\end{cases}\right.
$$

where $0<\tau<t^{*}, \Omega-$ maximum relative angular velocity of the disc. According with condition (15), the body begin to move if one of the following conditions satisfied:

$$
\tau \leq|\Omega| \frac{J_{d}}{T_{\varphi}}, \quad \text { or } \quad \tau \geq t^{*}-|\Omega| \frac{J_{d}}{T_{\varphi}} .
$$

On Figure 4 showed spaces of parameters $\left(\tau, t^{*}\right)$ (Figure $\left.4 \mathrm{a}\right)$ and $(\tau,|\Omega|)$ (Figure $4 \mathrm{~b}$ ). Shaded areas correspond to the values of the parameters at which the body begins to move. 


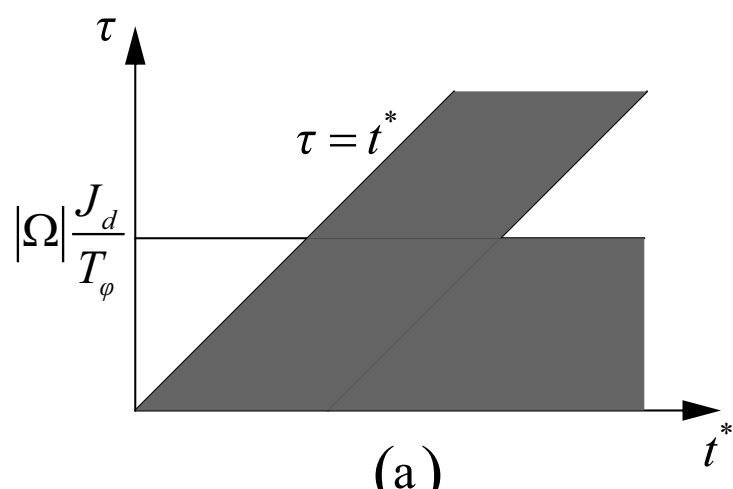

(a)

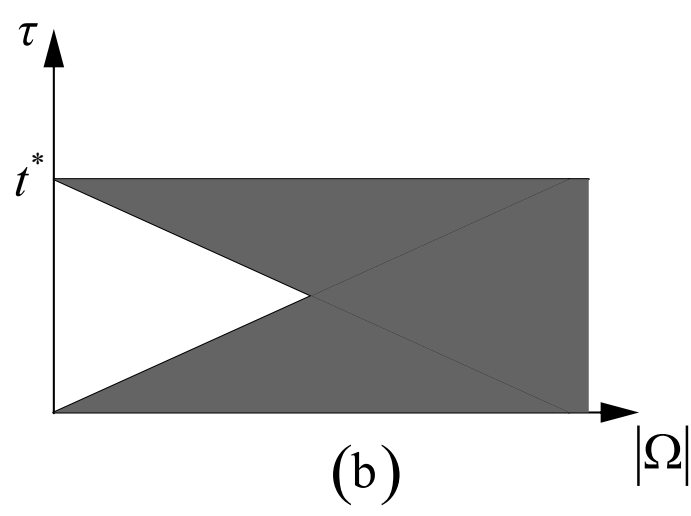

(b)

Figure 4: Shaded areas of the parameters $\left(\tau, t^{*}\right)$ (a) and $(\tau,|\Omega|)$ (b) corresponding beginning of the body movement

For the selected control law right part of the equation (14) piecewise constant. Depending on the functions $f_{A}$, equation takes the following three types:

$$
\ddot{\varphi}=0, \quad \ddot{\varphi}=\frac{R_{d}}{C+J_{d}}\left(1 \pm \frac{T_{\varphi}}{R_{d}}\right) .
$$

Thus the trajectory of the body $\varphi(t)$ for piecewise constant control of the relative acceleration of the disk consists of a set of lines and parabolas.

\subsection{Two point masses}

Let us place inside the body two material points with masses $m_{1}=m_{2}$. Mass can move relative to the body on two rails parallel to its longitudinal axis of symmetry and lying in a horizontal plane passing through the body center of mass $O$ (Figure $3 \mathrm{~b}$ ). The rails are positioned at equal distances $\eta_{1}=-\eta_{2}>0$ of the point $O$. We assume that the masses are moved inside the body in antiphase: $\xi_{1}(t)=-\xi_{2}(t)$. Kinetic moment of $i$-th point mass $(i=1,2)$ :

$$
\boldsymbol{K}_{O i}=\boldsymbol{r}_{O i} \times m_{1} \boldsymbol{v}_{i}=m_{1} \boldsymbol{r}_{O i} \times\left(\dot{\varphi} \boldsymbol{k} \times \boldsymbol{r}_{O i}+\dot{\xi}_{i}(t) \boldsymbol{e}_{1}\right)=m_{1}\left(\dot{\varphi} r_{O i}^{2}-\dot{\xi}_{i}(t) \eta_{i}\right) \boldsymbol{k},
$$

where $\boldsymbol{v}_{i}$ - absolute velocity of $i$-th internal mass. Because of selected restrictions on the function $\xi_{i}(t)$ kinetic moment will be the same for both masses. Summarizing, calculating the time derivative and projecting on the unit vector $\boldsymbol{k}$, we obtain:

$$
\sum_{i=1}^{s} \dot{\boldsymbol{K}}_{O i} \cdot \boldsymbol{k}=2 m_{1}\left(\left(\xi_{1}^{2}(t)+\eta_{1}^{2}\right) \ddot{\varphi}+2 \xi_{1}(t) \dot{\xi}_{1}(t) \dot{\varphi}-\ddot{\xi}_{1}(t) \eta_{1}\right) .
$$

Thus, substituting this formula in (13), we find the equation of the slider motion with two internal masses moves in antiphase:

$$
\left(C+2 m_{1}\left(\xi_{1}^{2}(t)+\eta_{1}^{2}\right)\right) \ddot{\varphi}=R_{p}-f_{A} T_{\varphi}
$$


where $R_{p}=-2 m_{1}\left(2 \xi_{1}(t) \dot{\xi}_{1}(t) \dot{\varphi}-\ddot{\xi}_{1}(t) \eta_{1}\right)$. The value of $f_{A}$ is defined similarly to the previous subsection:

$$
f_{A}= \begin{cases}\dot{\varphi}|\dot{\varphi}|^{-1}, & \dot{\varphi} \neq 0 \\ R_{p} T_{\varphi}^{-1}, & \dot{\varphi}=0,\left|R_{p}\right| \leq T_{\varphi} \\ R_{p}\left|R_{p}\right|^{-1}, & \dot{\varphi}=0,\left|R_{p}\right|>T_{\varphi}\end{cases}
$$

Absolute value of the relative acceleration $\ddot{\xi}_{1}(t)$ of the internal mass is needed to start rotation of the body is bounded below:

$$
\left|\ddot{\xi}_{1}(t)\right|>\frac{T_{\varphi}}{2 m_{1} \eta_{1}} .
$$

Let's consider two-phase piecewise linear function $\xi_{1}(t)$ as a control law of the internal mass relative motion. The function must satisfy the boundary conditions: $\xi_{1}(0)=-a_{1}$, $\xi_{1}(\tau)=a_{1}, \xi_{1}\left(t^{*}\right)=-a_{1}$, where $a_{1}$ - amplitude of the movable mass displacement inside the body. Prescribed conditions satisfies the following function:

$$
\xi_{1}(t)=a_{1} \cdot \begin{cases}2 t \tau^{-1}-1, & 0 \leq t<\tau \\ 1-2(t-\tau)\left(t^{*}-\tau\right)^{-1}, & \tau \leq t \leq t^{*}\end{cases}
$$

Thus for the relative velocity $\dot{\xi}_{1}(t)$ we obtain:

$$
\dot{\xi}_{1}(t)=2 a_{1} \cdot \begin{cases}\tau^{-1}, & 0 \leq t<\tau \\ \left(\tau-t^{*}\right)^{-1}, & \tau \leq t \leq t^{*}\end{cases}
$$

Relative acceleration of the internal mass is always equals to zero, except the moments $0, \tau$ and $t^{*}$, when the relative velocity changes abruptly:

$$
\ddot{\xi}_{1}(t)=\frac{2 a_{1}}{\tau} \delta(t)-\left(\frac{2 a_{1}}{\tau}+\frac{2 a_{1}}{t^{*}-\tau}\right) \delta(t-\tau)+\frac{2 a_{1}}{t^{*}-\tau} \delta\left(t-t^{*}\right), \quad 0 \leq t \leq t^{*},
$$

where $\delta(t)$ - delta-Dirac function. It is obvious that for the chosen unction $\xi_{1}(t)$ condition (17) will be executed at time $0, \tau$ and $t^{*}$ with any parameters of the control law. Stepwise change of the relative velocity of the movable mass means the presence of an impact in the system, which has a decisive influence on the dynamics of the body. The magnitude of the stepwise change of angular velocity is founded by integrating the equation of motion (16) for infinitely small period of time which includes an impact. Thus, at moments $0, \tau$ and $t^{*}$ the body has the instantaneous increment of angular velocity:

$$
\dot{\varphi}(t+\varepsilon)-\dot{\varphi}(t-\varepsilon)=\frac{2 m_{1} \eta_{1}}{C+2 m_{1}\left(\xi_{1}^{2}(t)+\eta_{1}^{2}\right)} \int_{t-\varepsilon}^{t+\varepsilon} \ddot{\xi}_{1}(t) d t, \quad \varepsilon \rightarrow 0, \quad t=0, \tau, t^{*} .
$$

In the remaining time points relative angular acceleration of the internal mass is zero and the function $R_{p}=-4 m_{1} \xi_{1}(t) \dot{\xi}_{1}(t) \dot{\varphi}, t \neq 0, \tau, t^{*}$. 


\section{CONCLUSIONS}

In this work we considered a rigid body based on a rough surface. The body has a hollow parallelepiped shape and could contain a movable masses. Masses are arranged in such way that the slider center of mass remained constant inside the body and coincide with the body center of mass. It was assumed that the initial velocity of the body center of mass is zero. Thus the position of the body is specified by the rotation angle relative to the initial position. In the work the local Amonton-Coulomb law was used. As a model of normal stress distribution in the contact area between the body and the surface dynamically consistent linear model was selected. It was shown that within the framework of this model, the distribution of normal stresses are constant. The equation of body motion with an arbitrary configuration of movable masses was obtained.

As example considered horizontal disk and two point masses moving in antiphase. Considered a two-phase piecewise constant control law of the relative acceleration of the disk. Area of the control law parameters which correspond to overcome the force of static friction and start the motion was found. Motion was qualitatively described. For two point masses proposed two-phase piecewise linear control law of shift points. It is established that the motion of the system is determined by the presence of impact resulting by stepwise changes of the mass relative velocity.

Author express thanks to N. N. Bolotnik for helpful comments. The work was supported by the basic part of the state tasks in the field of scientific activity No. 2014/120 "Investigation of the regularities in the dynamics of systems with friction and the development of mobile robots without external drivers" (research No. 2583) and the Russian Foundation for Basic Research (No. 14-01-00432).

\section{REFERENCES}

[1] Chernousko, F.L. Analysis and optimization of the motion of a body controlled by means of a moveable internal mass. J Appl Math Mech, 2006. Vol. 70, N 6. Pp. 819-842.

[2] Chernousko, F.L. The optimal periodic motions of a two-mass system in a resistant medium. // J Appl Math Mech, 2008. Vol. 72, N 2. Pp. 116-125.

[3] Chernousko, F.L. and Bolotnik, N.N. Mobile robots controlled by internal bodies movement. Tr. IMM UrO RAN, 2010. Vol. 16, N 5. Pp. 213-222. (in Russian)

[4] Volkova, L.Iu. and Iatsun, S.F. Control of the three-mass robot motion moving in a liquid medium. Nonlinear Dynamics, 2011. Vol. 7, N 4. Pp. 845-857. (in Russian)

[5] Iatsun, S.F. and Volkova, L.Iu. Simulation of dynamic modes of vibration robot moving along the surface of the viscous resistance. Specialized machinery and communication, 2012. N 3. Pp. 25-29. (in Russian) 
[6] Ivanov, A.P. and Sakharov, A.V. On the dynamics of a rigid body with moving masses and a rotor on a rough plane. Nonlinear Dynamics \& Mobile Robotics, 2013. Vol. 1, N 1. Pp. 23-32.

[7] Sakharov, A.V. Rotation of a body without external drivers by a rotor. Proceedings of MIPT, 2014. Vol. 6, N 2. Pp. 80-91. (in Russian)

[8] Ivanov, A.P. Fundamentals ot the theory of systems with friction. Moscow-Izhevsk: Regular \& Chaotic Dynamics, Institute of Computer Sciences, 2011. 304 p. (in Russian)

[9] Jellett, J.H. A treatise on the theory of friction. Dublin, London: MacMillan, 1872. $220 \mathrm{p}$.

[10] Ivanov, A.P. A Dynamically consistent model of the contact stresses in the plane motion of a rigid body. J Appl Math Mech, 2009. Vol. 73, N 2. Pp. 134-144. 